\title{
Decisiveness indices are semiindices
}

\author{
Josep Freixas and Montserrat Pons*
}

\begin{abstract}
In this note we prove that any decisiveness index, defined for any voter as the probability of him/her being decisive, is a semiindex when the probability distribution over coalitions is anonymous, and it is a semiindex with binomial coefficients when the probability over coalitions is anonymous and independent.
\end{abstract}

Key words: game theory; voting; simple games; power indices; decisiveness. Math. Subj. Class. (2000): 91A12, 91A80. JEL Class.: C71, D72.

\section{Preliminaries}

\subsection{Simple game context}

Assume that a proposal is put to the vote, that it must be either approved or rejected and that each voter can only vote "yes" or "no". A model for this binary voting scenario is a simple game $(N, \mathcal{W})$, where $N=\{1,2, \ldots, n\}$ denotes the set of voters and $\mathcal{W}$ denotes the set of winning coalitions. A coalition is any subset $S \subseteq N$, and is interpreted as the set of voters which vote "yes". A coalition is winning when its occurrence causes the proposal to be accepted. For any coalition $S$ we denote $s=|S|$. When the set of voters, $N$, is fixed we will write $\mathcal{W}$ instead of $(N, \mathcal{W})$.

Let $\mathcal{S}_{N}$ be the set of simple games on $N$. A power index on $\mathcal{S}_{N}$ is a map $\psi: \mathcal{S}_{N} \rightarrow \mathbb{R}^{n}$ that assigns to every simple game $\mathcal{W}$ a vector $\psi(\mathcal{W})$ with components $\psi_{i}(\mathcal{W})$ for all $i \in N$.

Many power indices have been considered in the literature. Most of them are based on the notion of cruciality. If $\mathcal{W}$ is a simple game, a player $i \in N$ is said to be crucial for a coalition $S \subseteq N \backslash\{i\}$ if $S \notin \mathcal{W}$ but $S \cup\{i\} \in \mathcal{W}$. We then write $S \in \mathcal{C}_{i}(\mathcal{W})$. This is equivalent to say that the marginal contribution of player $i$ to $S \cup\{i\}$ in $\mathcal{W}$ is 1 . Intuitively, any measure of power should take account of the times each player is crucial in a game. Let's recall a family of power indices of this type, named semiindices. These power indices were first introduced by Weber [8] on simple games and extended to all cooperative games by Dubey et al [4].

A semiindex is a power index on $\mathcal{S}_{N}$ that satisfies the well-known axioms of: symmetry, positivity, dummy player property and transfer. The following characterization of semiindices by means of coefficients was established in Carreras et al. [3], following the characterization given in Dubey et al. [4]:

*The authors are with the Universitat Politècnica de Catalunya (Barcelona-Tech), in the Department of Mathematics; e-mails: josep.freixas@upc.edu, montserrat.pons@upc.edu; postal address: EPSEM, Avda. Bases de Manresa, 61-73, E-08242 Manresa, Spain. 
(a) for every $\mathbf{p}=\left(p_{1}, \ldots, p_{n}\right)$ such that $\sum_{s=1}^{n} p_{s}\left(\begin{array}{c}n-1 \\ s-1\end{array}\right)=1$ and $p_{s} \geq 0$ for all $s$, the expression

$$
\mathfrak{S}_{i}^{\mathbf{p}}(\mathcal{W})=\sum_{S \in \mathcal{C}_{i}} p_{s}
$$

where $s=|S|$, defines a semiindex $\mathfrak{S}^{\mathbf{p}}$ on $\mathcal{S}_{N}$;

(b) conversely, every semiindex on $\mathcal{S}_{N}$ can be obtained in this way;

c) the correspondence given by $\left\{p_{k}\right\} \mapsto \mathfrak{S}^{\mathbf{p}}$ is bijective.

Thus, the payoff that a semiindex allocates to every player in any game is a weighted sum of his/her marginal contributions in the game. Well known examples of semiindices are: the Shapley-Shubik power index $\varphi$ (Shapley and Shubik [7]), for which $p_{s}=1 /\left[n\left(\begin{array}{l}n-1 \\ s-1\end{array}\right)\right]$ for any $s(1 \leq s \leq n)$, and the Banzhaf power index $\beta$ (Banzhaf [1] and Penrose [6]) for which $p_{s}=1 / 2^{n-1}$. The Shapley and Shubik power index is the only efficient semiindex in the sense that $\sum_{i \in N} \varphi_{i}(\mathcal{W})=1$ for every game $\mathcal{W}$. The Banzhaf power index is the only semiindex having constant coefficients. A subclass of semiindices, that contains the Banzhaf index, is formed by those with binomial coefficients: $p_{s}=t^{s-1}(1-t)^{n-s}$ for some $t \in[0,1]$, introduced by Carreras and Freixas [2].

From now on we will denote by $\mathfrak{S}^{\mathbf{p}}$ the semiindex uniquely defined by the vector $\mathbf{p}$ in (1) and by $\mathfrak{B}^{t}$ the particular case of semiindex with binomial coefficients:

$$
\mathfrak{B}_{i}^{t}(\mathcal{W})=\sum_{S \in \mathcal{C}_{i}} t^{s-1}(1-t)^{n-s}
$$

\subsection{Simple games with probability distributions over coalitions}

A probability distribution over coalitions is a function $P: 2^{N} \rightarrow \mathbb{R}$ such that $P(S) \geq 0$ for all $S \subseteq N$ and $\sum_{S \in 2^{N}} P(S)=1 . P(S)$ is interpreted as the probability that voters in $S$ vote "yes" and voters is $N \backslash S$ vote "no".

Let $\mathcal{P}_{N}$ be the set of probability distributions over coalitions on $N$. For any $P \in \mathcal{P}_{N}$, a decisiveness index can be defined on $\mathcal{S}_{N}$, as pointed out by Dubey and Shapley in [5]. The decisiveness index we are considering captures the idea that voters become crucial for a given coalition by either changing its status from winning to losing or conversely, and it measures the probability of a voter being decisive.

Given $P \in \mathcal{P}_{N}$ then, for any voter $i \in N$, the decisiveness index is defined as:

$$
\Phi_{i}^{P}(\mathcal{W})=\sum_{\substack{S: i \in S \in \mathcal{W} \\ S \backslash\{i\} \notin \mathcal{W}}} P(S)+\sum_{\substack{S: i \notin S \notin \mathcal{W} \\ S \cup\{i\} \in \mathcal{W}}} P(S)=\sum_{S \in \mathcal{C}_{i}}[P(S)+P(S \backslash\{i\})]
$$

The Banzhaf index $\beta$ and the Shapley-Shubik index $\varphi$ are examples of decisiveness indices for particular probability distributions. Indeed, $\beta=\Phi^{P^{*}}$ and $\varphi=\Phi^{P^{* *}}$, where $P^{*}(S)=1 / 2^{n}$ and $P^{* *}(S)=1 /\left[(n+1)\left(\begin{array}{l}n \\ s\end{array}\right)\right]$ for all $S \subseteq N$. 
We center now our attention in anonymous probability distributions.

A probability distribution $P$ is:

i) anonymous if $P(S)=P(T)$ whenever $|S|=|T|$,

ii) independent if every voter's vote is independent of the others. In this case, if $t_{i}$ is the probability of $i$ voting "yes":

$$
P(S)=\prod_{i \in S} t_{i} \prod_{i \notin S}\left(1-t_{i}\right)
$$

Consequently in an anonymous and independent probability distribution every voter casts the vote independently of the other voters with the same probability $t_{i}=t$ for all $i \in N$ so that

$$
P(S)=t^{s}(1-t)^{n-s}
$$

Note that the Banzhaf index is obtained from a probability distribution $P^{*}$ which is both, anonymous and independent, with $t_{i}=1 / 2$ for all $i \in N$. The Shapley-Shubik index is obtained from the probability distribution $P^{* *}$ which is anonymous but not independent.

The purpose of this note is to show that the decisiveness indices $\Phi^{P}$ are closely related to semiindices when the probability distribution $P$ is anonymous and this relation is still greater when it is anonymous and independent.

\section{Decisiveness indices for anonymous probability distributions}

Let $\mathcal{A}_{N} \subseteq \mathcal{P}_{N}$ denote the set of anonymous probability distributions. If $P \in \mathcal{A}_{N}$ denote $P_{s}=P(S)$ for all $S \subseteq N$. Then the decisiveness index in (3) reduces to

$$
\Phi_{i}^{P}=\sum_{S \in \mathcal{C}_{i}}\left[P_{s}+P_{s-1}\right]
$$

with $\sum_{s=0}^{n}\left(\begin{array}{l}n \\ s\end{array}\right) P_{s}=1, P_{s} \geq 0$ for all $s=0,1, \ldots, n$.

Proposition 2.1 Let $P \in \mathcal{A}_{N}$. Then the decisiveness index $\Phi^{P}$ coincides with the semiindex $\mathfrak{S}^{\mathbf{p}}$ defined in (1) with coefficients $p_{s}=P_{s}+P_{s-1}$ for all $s=1, \ldots, n$.

Proof: We have to prove that the vector $\mathbf{p}=\left(p_{1}, \ldots, p_{n}\right)$ defined by $p_{s}=P_{s}+P_{s-1}$ for all $s=1, \ldots, n$ determines a semiindex, i.e., $p_{s} \geq 0$ for all $s=1, \ldots, n$ and $\sum_{s=1}^{n}\left(\begin{array}{c}n-1 \\ s-1\end{array}\right) p_{s}=1$. Then, from (1) and (6), we deduce that $\Phi^{P}=\mathfrak{S}^{\mathbf{p}}$.

It is clear that $p_{s} \geq 0$ for $s=1, \ldots, n$ because $P_{s} \geq 0$ for all $s=0,1, \ldots, n$.

Using that $\sum_{s=0}^{n}\left(\begin{array}{l}n \\ s\end{array}\right) P_{s}=1$ we deduce that $\sum_{s=1}^{n}\left(\begin{array}{c}n-1 \\ s-1\end{array}\right) p_{s}=1$ as follows: 


$$
\begin{aligned}
& \sum_{s=0}^{n}\left(\begin{array}{l}
n \\
s
\end{array}\right) P_{s}=1 \quad \Longleftrightarrow \quad P_{0}+\sum_{s=1}^{n-1}\left(\begin{array}{l}
n \\
s
\end{array}\right) P_{s}+P_{n}=1 \\
\Longleftrightarrow & P_{0}+\sum_{s=1}^{n-1}\left[\left(\begin{array}{c}
n-1 \\
s-1
\end{array}\right)+\left(\begin{array}{c}
n-1 \\
s
\end{array}\right)\right] P_{s}+P_{n}=1 \quad \Longleftrightarrow \quad P_{0}+\sum_{s=1}^{n-1}\left(\begin{array}{c}
n-1 \\
s-1
\end{array}\right) P_{s}+\sum_{s=2}^{n}\left(\begin{array}{c}
n-1 \\
s-1
\end{array}\right) P_{s-1}+P_{n}=1 \\
\Longleftrightarrow & \left(P_{0}+P_{1}\right)+\sum_{s=2}^{n-1}\left(\begin{array}{c}
n-1 \\
s-1
\end{array}\right) P_{s}+\sum_{s=2}^{n-1}\left(\begin{array}{c}
n-1 \\
s-1
\end{array}\right) P_{s-1}+\left(P_{n-1}+P_{n}\right)=1 \quad \Longleftrightarrow \quad \sum_{s=1}^{n}\left(\begin{array}{c}
n-1 \\
s-1
\end{array}\right)\left(P_{s}+P_{s-1}\right)=1 \\
\Longleftrightarrow & \sum_{s=1}^{n}\left(\begin{array}{c}
n-1 \\
s-1
\end{array}\right) p_{s}=1
\end{aligned}
$$

Nevertheless, not every semiindex comes from a decisiveness index. As an example consider for $n=3$ the semiindex $\mathfrak{S}^{\mathbf{p}}$ defined by $\mathbf{p}=\left(p_{0}, p_{1}, p_{2}\right)=(1 / 8,3 / 8,1 / 8)$. In fact, if there were $P \in \mathcal{A}_{N}$ satisfying the three equalities in Proposition 2.1 then it should be $P_{1} \leq 1 / 8$ and $P_{2} \leq 1 / 8$ from the first and third equalities, which would contradict the second equality $P_{1}+P_{2}=3 / 8$. The characterization of the semiindices which come from a decisiveness index is an open problem.

A direct consequence of Proposition 2.1 is the following corollary, which establishes a bijective correspondence between the semiindices with binomial coefficients defined in (2) and the decisiveness indices given by anonymous and independent probability distributions. Let $\mathcal{I}_{N} \subseteq \mathcal{A}_{N}$ denote the set of anonymous and independent probability distributions.

Corollary 2.2 If $P \in \mathcal{I}_{N}$ then $\Phi^{P}=\mathfrak{B}^{t}$ for some $t \in[0,1]$, and, conversely, any semiindex $\mathfrak{B}^{t}$ can be obtained in this way.

Proof: From (5), if $P \in \mathcal{I}_{N}$ then there exist some $t \in[0,1]$ such that $P_{s}=t^{s}(1-t)^{n-s}$, for $s=0,1, \ldots, n$. From Proposition 2.1 we know that $\Phi^{P}=\mathfrak{S}^{\mathbf{p}}$ with the vector $\mathbf{p}=\left(p_{1}, \ldots, p_{n}\right)$ defined by $p_{s}=P_{s}+P_{s-1}$ for all $s=1, \ldots, n$. Thus, $p_{s}=t^{s}(1-t)^{n-s}+t^{s-1}(1-t)^{n-s+1}=$ $t^{s-1}(1-t)^{n-s}[t+(1-t)]=t^{s-1}(1-t)^{n-s}$, and these coefficients define the semiindex $\mathfrak{B}^{t}$. As this proof is independent of $t$ we can conclude that any semiindex with binomial coeficients is a decisiveness index $\Phi^{P}$ with $P \in \mathcal{I}_{N}$.

\section{Acknowledgements}

This research was partially supported by Grant MTM2012-34426/FEDER from "Ministerio de Economía y Competitividad" of the Spanish Government.

\section{References}

[1] J.F. Banzhaf. Weighted voting doesn't work: a mathematical analysis. Rutgers Law Review, 19(2):317-343, 1965. 
[2] F. Carreras and J. Freixas. Some theoretical reasons for using regular semivalues. In H. De Swart, editor, Logic, Game Theory and Social Choice. Proceedings of the International Conference, LGS, pages 140-154, Tilburg, the Netherlands, 1999.

[3] F. Carreras, J. Freixas, and M.A. Puente. Semivalues as power indices. European Journal of Operational Research, 149:676-687, 2003.

[4] P. Dubey, P. Neyman, and R.J. Weber. Value theory without efficiency. Mathematics of Operations Research, 6:122-128, 1981.

[5] P. Dubey and L.S. Shapley. Mathematical properties of the Banzhaf power index. Mathematics of Operations Research, 4(2):99-131, 1979.

[6] L.S. Penrose. The elementary statistics of majority voting. Journal of the Royal Statistical Society, 109(1):53-57, 1946.

[7] L.S. Shapley and M. Shubik. A method for evaluating the distribution of power in a committee system. American Political Science Review, 48(3):787-792, 1954.

[8] R.J. Weber. Subjectivity in the valuation of games. In O. Moeschlin and D. Pallaschke, editors, Game theory and related topics, pages 129-136. North Holland Publishing Co., Amsterdam, 1979. 